\title{
NUMERICAL METHOD FOR ESTIMATION OF TENSILE LOAD IN TIE-RODS
}

\author{
Ksenita Riabova*, Luca Collini, Rinaldo Garziera \\ University of Parma, Parco Area delle Scienze, 181/A, Parma, Italy \\ * corresponding author: kseniia.riabova@studenti.unipr.it
}

\begin{abstract}
The work introduces a two-phase method for determination of axial loads in tie-rods. The method described here consists of an experimental activity and an automated numerical calculation. The influence of considering an elastic Winkler-type bed to model the tie-rod constraint inside the wall has been investigated. The algorithm used for calculation involves a solution of a functional minimization problem, where the tensile load and the stiffness of elastic foundation at the edges are used as optimization parameters and the error function, which describes the deviation between the frequencies measured and those calculated using finite element method, is minimized. Qualitative analysis of the results showed a significant reduction of the error compared to models with different boundary conditions. The method showed to be conservative for the strength evaluation of the rods, because the optimal values of tensile loads appeared to be higher than the load in perfect encastre conditions.
\end{abstract}

KEYwords: tie-rods, non-destructive technique, numerical method.

\section{INTRODUCTION}

Tie-rods are structural elements used in a wide range of civil constructions. One of purposes they serve is to provide support for masonry arches and vaults in ancient buildings (e.g. churches, castles, etc.), which are known to lurch and founder course of time. Tierods are subjected to axial tension and, thus, help the building resist occurring lateral loads exerted by structural elements, walls and facades.

Over the years deformation of masonry walls and some displacements in the building may cause significant changes in the axial loads of tie-rods. In the extremes this can lead to either failures in tie-rods structural integrity, or to laziness of tie-rods that stop carrying out their duty when they loose loads. That is why regular monitoring of tie-rods condition is of a great importance.

The goal of the presented work is to determine axial loads acting on the tie-rods of the fifteenth century Casa Romei located in Ferrara, Emilia-Romagna, Italy, and thus to evaluate their functionality and reliability. An approach based on an experimental investigation and a numerical method is introduced to serve this purpose.

\section{Previous Studies}

Some attempts have been made throughout the years to develop an appropriate non-destructive technique for and indirect estimation of the tensile load in tierods. Previous studies [1 3] report a method that combined static and dynamic force identification. Tierods were modeled as simply supported Euler beams with rotational springs of similar stiffness added on each edge. The stiffness of the spring and the force were the two unknowns obtained from the system of equations, built with a static equation for deflection and a dynamic equation for natural frequencies. Another study [4] introduced a static approach for force identification. The experiment consisted of measuring three vertical displacements and strains variations at three sections of the tie-rod under a concentrated load; in [5] an algorithm to identify the axial tensile force in ancient tie-rods by using the first three natural frequencies is developed. The tie-rod was modelled as an Euler beam of uniform cross-section, neglecting the shear deformation and rotary inertia, and was assumed to be simply supported at the ends with additional rotational springs.

Recently, Maes et al. 6] introduced a method that enables definition of axial loads in slender beams with unknown boundary conditions, taking into account affects of rotational inertia of the beam and masses of sensors. However, it requires data from five or more sensors along the length of the beam to determine all the introduced unknown of the inverted problem. A similar technique of the axial force identification was developed by Li et al. [7 focusing on studies of Euler-Bernoulli beams and takes into account bending stiffness effects. Rebecchi et al. [8] established an analytical method of processing experimental data from five instrumented sections of a prismatic slender beam, which showed excellent results in estimation of the axial load in tie-rods. The method does not require any exact value of effective length of the beam, but neglects both rotary inertia and shear deformations effects in the solution for beam vibrations. For cases of similar beams, Tullini et al. 911 proposed a static method of axial force identification. The analytical algorithm makes use of any set of experimental data 
represented by flexural displacements or curvatures measured at five cross-sections of the beam subjected to an additional concentrated lateral load. Again, Gentilini et al. [12 developed a procedure that combines dynamical testing with FEM simulations using added masses. The method was tested out for tie-rods of various length and load intensity, showing reliable results. Livingston et al. [13] identified the tensile force in prismatic beams of uniform section by using modal data and assuming rotational and vertical springs at each end of the beam. Shear deformation and rotary inertia were neglected (according to the Euler beam model).

\section{Estimation Method}

In the present study authors intend to introduce a method of health monitoring of tie-rods inserted in ancient monumental masonry buildings. The method consists of an in-situ experimental activity and an automated two-parameter optimization algorithm which allows to evaluate the axial load in tie-rods with a good precision. The technique is basically a simply executed frequency-based identification method that allows to minimize the estimation error.

About six natural frequencies can be easily identified with a simple test by experimentally measuring the frequency response functions (FRFs) of the tierods with instrumented hammer excitation. Further a parametric finite element model of a tie-rod is developed in the FEM analysis software Abaqus.

The part of a tie-rod hidden inside the masonry wall is assumed to have a length of $0.2 \mathrm{~m}$ and the constraints given to this part are modelled as elastic foundations (Winkler bed). The tensile force and the stiffness of the foundation are chosen as the unknown parameters. In some cases the length of the rod inserted inside the masonry wall can be also assumed as unknown. The sought axial load, as it is explained subsequently, is obtained through an optimization routine that minimizes the deviation between corresponding experimentally determined eigenfrequencies and those calculated by FEM.

The authors have previously tested the present technique with some variations over different buildings and situations [14-16. The novelty introduced in the present work is the optimization with respect to the stiffness of the Winkler bed type boundary conditions.

\subsection{Experimental Part}

The tie-rods investigated during the case study have been installed at different points along the lifespan of Casa Romei (see Fig. 1), therefore they differ in dimensions, cross-section shapes and material condition. Measurements of geometrical characteristics and of natural frequencies were performed for each reachable tie-rod of the building. The natural frequencies were obtained from the analysis of response to dynamical excitation applied to tie-rods in horizontal plane for a more accurate estimation of the axial load. Since

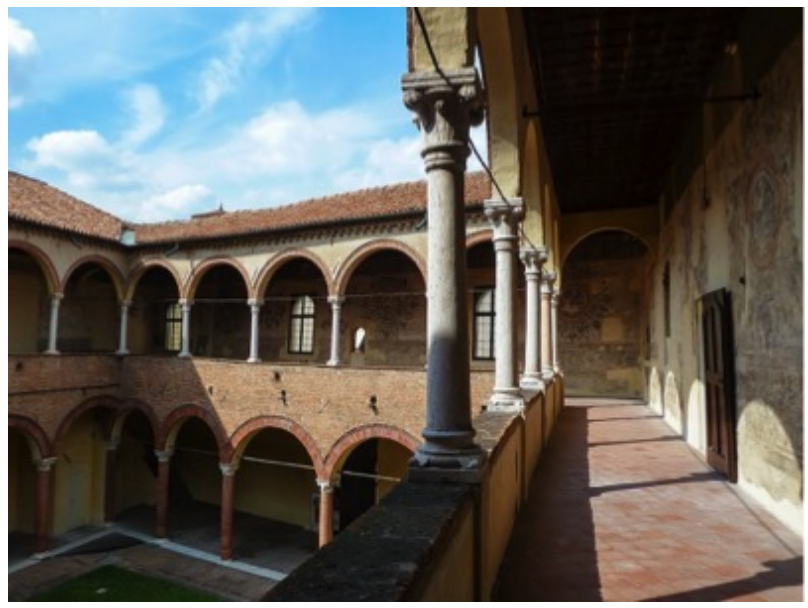

FIGURE 1. Inner yard of Casa Romei.

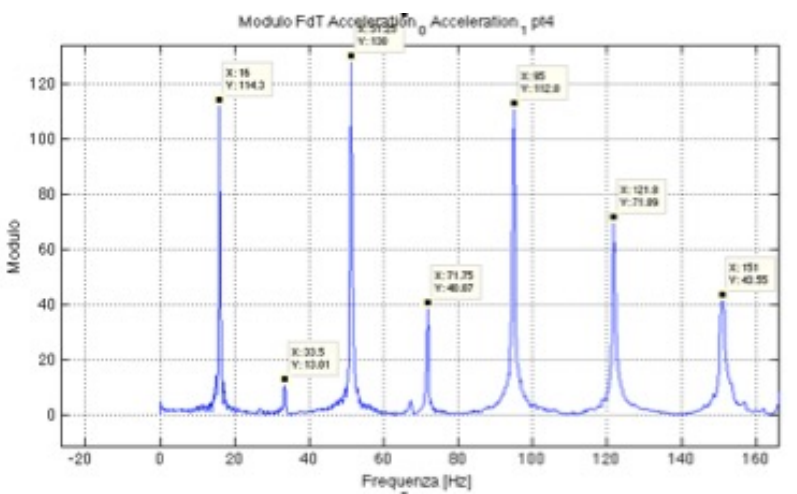

Figure 2. A typical FRF plot (acceleration amplitude vs. frequency).

the studied rods are much stiffer in the vertical plane, tests in vertical direction were not performed.

The used instrumentation was composed by tools listed in Tab. 1

The signal from accelerometers was handled by the dynamic signal acquisition module National Instrument (NI) 9234 and acquisition software developed in LabView and further elaborated in MatLab resulting in the frequency response functions (FRF) for each tierod. FRF for a ground floor tie-rod Pt4 is presented in Fig. 2,

Another important part of the experimental activity was to measure accurately the geometric characteristics of tie-rods, i.e. cross-section dimensions and lengths. For the further study it was assumed that the cross-section remains the same along all the length of a rod. The tested rods are crafted in iron for which the characteristics vary insignificantly, thus the material data was assumed as follows: $E=2 \times 10^{11} \mathrm{~N} / \mathrm{m}^{2}$, $\rho=7850 \mathrm{~kg} / \mathrm{m}^{3}, v=0.3$. The main parameters $E$ and $v$ appear in the frequencies under the square root, so even some variation in these values can only have a minor influence on the natural frequencies.

For the further analysis first six natural frequencies were identified for each tie-rod by means of peakpicking from FRFs. Six eigenmodes are providing 


\begin{tabular}{cccc}
\hline Tool & Manufacturer & Model & Sensitivity \\
\hline Impact Hammer & Brï£jel\&Kjaer & 8202 & $4 \mathrm{pc} / \mathrm{N}$ \\
\hline Accelerometer & PCB & $356 \mathrm{~A} 01$ & $0,01 \mathrm{~V} /\left(\mathrm{m} / \mathrm{s}^{2}\right)$ \\
\hline Accelerometer & PCB & $356 \mathrm{~A} 01$ & $0,01 \mathrm{~V} /\left(\mathrm{m} / \mathrm{s}^{2}\right)$ \\
\hline
\end{tabular}

TABLE 1. List of experimental tools.

an over constrained problem, thus the higher modes were not taken under consideration in this research. Besides that, identification of higher modes might appear inaccurate due to larger possible measurement errors.

\subsection{NUMERICAL PART}

Since the extremities of tie-rods are built into the masonry walls in a way that hardly leaves a possibility to be certain about the end constraints, as a first iteration, boundary conditions for the edges were modeled as plain fixed and/or simply supported. In both cases for any range of optimization parameters (length and force) and weight coefficients the error function reaches minimum every time for the minimal length in the range, i.e. for the measured length. This means that the minimum is forced. Investigation of the function in symbolic mathematics package Maple 13.0 showed good correspondence of the results obtained from FEM with analytical models of a string and a beam under axial load. Also the error function has an absolute minimum that lies however below the length measured. This kind of behavior proves that the BCs are irrelevant.

The real conditions on the edges of tie-rods are unknown and may vary from one rod to another, because they are fixed inside masonry walls in a way that is hard to determine using non-destructive techniques. However, certain attempts can be made in order to develop a generalized parametric model suitable for numerical determination of loads in tie-rods.

In order to determine the loads, tie-rods are modeled in FEM software Abaqus using 3D beam elements that incorporate Timoshenko beam theory (B31), which allows to take into account shear deformation and rotational inertia effects. The cross section is assumed to be constant along each rod.

As a first step a pretension load $\mathrm{N}$ is applied to the beam and as a second step modal analysis is performed. Since the value of the pretension load is the subject of search, it has to be tuned to make the results of modal analysis in FEM reach a good agreement with experimentally defined frequencies. However, manual tuning of the load is not considered to be an option in this case, because there is no certainty about the boundary conditions at the beam edges.

This problem is overcome by means of a parametric FEM model and an optimization tool for computing. In FEM model the measured length of the tie-rod is represented by 50 beam elements; boundary condi- tions are assumed to be an elastic bed and, thus, are represented by 5 spring elements on each edge of the beam, acting in the direction of considered vibrations. Also the displacement in direction $\mathrm{X}$ (tie-rod axis) is restrained. The sketch of the assumed model is displayed in Fig. 3 .

Stiffness of each spring element together with the sought load are chosen as optimization parameters in the problem stated. The optimization program requires a set of experimentally obtained frequencies, ranges of stiffness and force with sizes of steps for each and also a set of weight coefficients for frequencies, that define importance of the corresponding frequency for the analysis. Combinations of parameters form a grid and the program launches ABAQUS input file for each nod of this grid and then extracts and filters natural frequencies. Vibrations in $\mathrm{Z}$ direction only are of our particular interest due to testing that was carried out in the horizontal plane. Optimization criteria for the problem is represented by the residual error between experimentally defined frequencies and those obtained from FEM analysis. The error $E$ is calculated according to the following formula:

$$
E=\sqrt{\sum_{k=1}^{n} p_{k}^{2}\left(f_{k}^{e x p}-f_{k}^{F E M}\right)^{2}}
$$

where $p_{k}$ is a weight arbitrary given to each frequency. The optimal values of stiffness of the elastic bed and of axial force deliver minimum value of the error function.

The optimization process starts from a reasonable value of the load which can be predicted as an optimal load for a simplified model with fixed ends, i.e. for infinite stiffness of the elastic bed at the constraints. The second step to be done is now to vary the stiffness of the spring bed, and optimize their value to minimize again the error. This implies a refinement process of the parameters along what we call zooming technique [15. Operatively, the algorithm defines a matrix of values and refines grid of parameters to be solved just where local minima of the error as defined in Eq. 1] are found.

A typical graphic of the surfaces representing the error distribution is presented in Eq.4. Here the error values are plotted with respect to stiffness and axial load, for a specific tie-rod. The free length $L$, and length of $L_{f}$ (in Fig. 3 ) are kept constant. The clear minima of the error functional can be observed. 


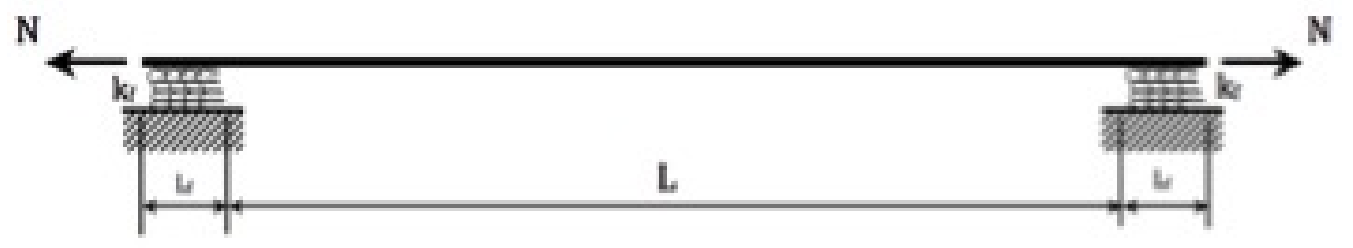

FIGURE 3. Sketch of the computational model.

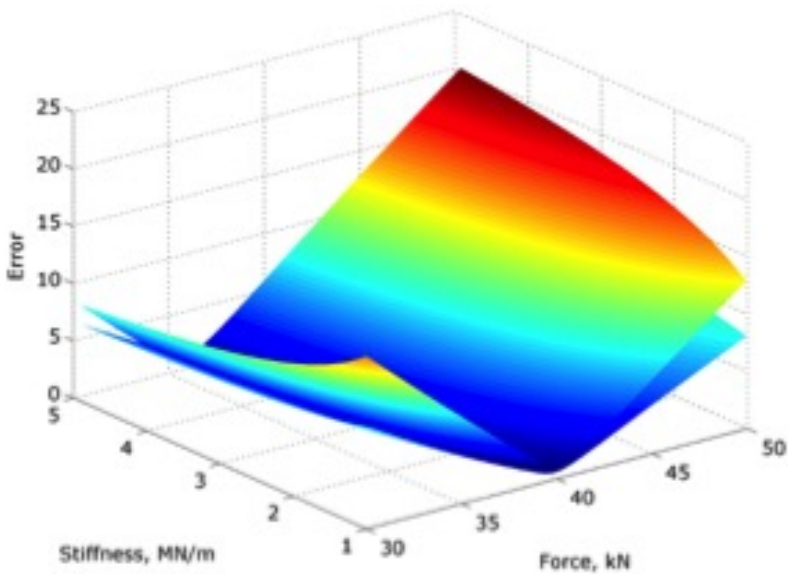

FiguRE 4. Error as function of bed stiffness and axial load for different sets of weight coefficients.

The error which represents the difference between numerical and experimental frequencies is calculated from the Eq. 1. Each of the differences (in $\mathrm{Hz}$ ) between couples of frequencies is multiplied by a weight $p_{k}$ in order to allow us to assign higher (or lower) importance to some frequencies rather than the others. The set of weights is then arbitrary chosen, but generally, the first frequencies, or just the fundamental one, have greater importance. A sensitivity analysis showed that the minima of the error functional Eq. 1 with respect to the sought tensile load is not influenced by the weights set (see Fig. 4).

The variation of the error is depicted in Fig. 5 , wherefrom it can be observed that the minimum value of the error is reached for the optimal elastic bed stiffness and is reached for the up to higher load compared to the encastre boundary conditions, thus, the method reveals to be conservative for the strength evaluation of the rods.

The values of error vary from one tie-rod to another, this shows that the model with Winkler bed boundary conditions fits better for some of the rods, however these errors were less than those calculated for ordinary fixed or simply supported boundary conditions. For a couple of tie-rods though a perfect encastre boundary condition at the walls appeared to be the best approximation which was demonstrated by an improvement in the error value compared to the case when a set of springs was modelled at their extremities.

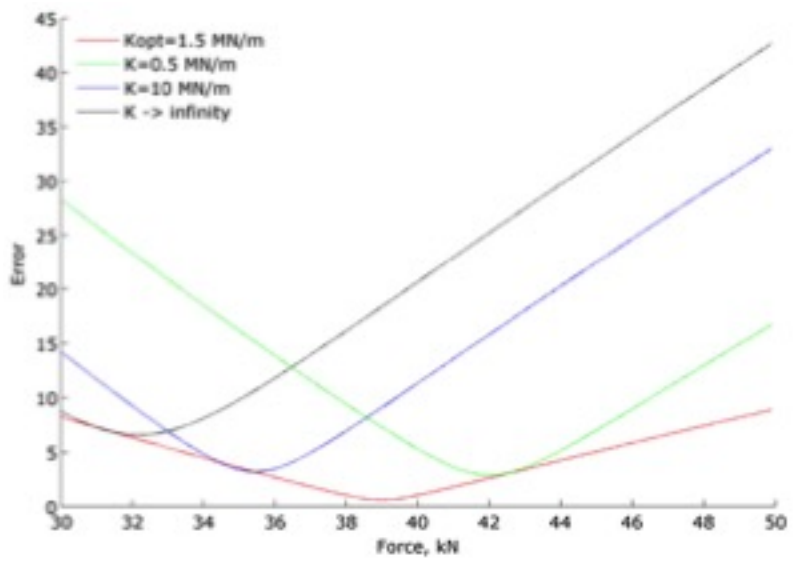

FiguRE 5. Influence of the elastic bed stiffness on the minimal error.

\section{Conclusions}

In this work an optimization process is shown and discussed in determining the axial load in structural iron tie-rods. The influence of considering an elastic Winkler-type bed to model the tie-rod constraint into the wall has been investigated. This constitutes the major novelty of this work, with respect to the previous studies.

Structural assessment of the overall building stability is enabled by the described method throughout the identification of axial loads in tie-rods via experimental and numerical activity. In particular, the introduced computational model is able to take into account even unknown boundary conditions that are typical for tie-rods installed in ancient buildings. Furthermore, from the simulations it was concluded that the variation of tensile load shifts the set of frequencies (higher the load, higher the frequencies), while the change in elastic bed stiffness changes the distance between natural frequencies. And finally, the conclusion about the method convergence can also be made based on the fact that different sets of weight coefficients bring to minor changes in the sought axial load in tie-rods.

\section{REFERENCES}

[1] C. Blasi. Sulla determinazione del "tiro" nelle catene mediante prove statiche e dinamiche. Atti III Congresso Nazionale ASSIRCO 1988.

[2] C. Blasi. Determining the axial force in metallic rods. Structural Engineering International (IABSE) 4:241-246, 1994. 
[3] S. Sorace. Parameter models for estimating in-situ tensile force in tie-rods. Journal of Engineering Mechanics 122:818-825, 1996.

[4] S. Briccoli Bati. Experimental method for estimating in situ tensile force in tie-rods. Journal of Engineering Mechanics 127:1275-1283, 2001.

[5] S. Lagomarsino. The dynamical identification of the tensile force in ancient tie-rods. Engineering Structures 27:846-856, 2005.

[6] K. Maes. Identification of axial forces in beam members by local vibration measurements. Journal of Sound and Vibration 332:5417-5432, 2013. DOI:10.1016/j.ijrmms.2008.06.005

[7] S. Li. Vibration-based estimation of axial force for a beam member with uncertain boundary conditions. Journal of Sound and Vibration 332:795-806, 2013.

[8] G. Rebecchi. Estimate of the axial force in slender beams with unknown boundary conditions using one flexural mode shape. Journal of Sound and Vibration 332:4122-4135, 2013. DOI:10.1016/j.jsv.2013.03.018.

[9] N. Tullini. Dynamic identification of beam axial loads using one flexural mode shape. Journal of Sound and Vibration 318:131-147, 2008.
[10] N. Tullini. Bending tests to estimate the axial force in tie-rods. Mechanics Research Communications 44:57-64, 2012.

[11] N. Tullini. Bending tests to estimate the axial force in slender beams with unknown boundary conditions. Mechanics Research Communications 53:15-23, 2013.

[12] C. Gentilini. Nondestructive characterization of tie-rods by means of dynamic testing, added masses and genetic algorithms. Journal of Sound and Vibration 332:76-101, 2013. DOI:10.1016/j.jsv.2012.08.009.

[13] T. Livingston. Estimation of axial load in prismatic members using flexural vibrations. Journal of Sound and Vibration 179:899-908, 1995. DOI:10.1016/j.jsv.2012.10.019

[14] M. Amabili. Estimation of tensile force in tie-rods using a frequency-based identification method. Journal of Sound and Vibration 329:2057-2067, 2010.

[15] M. Amabili. A hybrid method for the nondestructive evaluation of the axial load in structural tie-rods. Nondestructive Testing and Evaluation 26(2):197-208, 2011.

[16] L. Collini. Load and effectiveness of the tie-rods of an ancient dome: Technical and historical aspects. Journal of Cultural Heritage 16:597-601, 2015. 